\title{
Exosome-mediated peritoneal dissemination in gastric cancer and its clinical applications (Review)
}

\author{
KAI-BO CHEN ${ }^{*}$, JIAN CHEN* ${ }^{*}$ XIAO-LI JIN, YI HUANG, QIU-MING SU and LI CHEN \\ Department of General Surgery, The Second Affiliated Hospital, College of Medicine, \\ Zhejiang University, Hangzhou, Zhejiang 310000, P.R. China
}

Received January 30, 2018; Accepted April 11, 2018

DOI: $10.3892 /$ br.2018.1088

\begin{abstract}
The prognosis of patients with peritoneal dissemination from gastric cancer is poor, and the underlying molecular mechanism remains unclear. Exosomes, as macromolecular phospholipid bilayer vesicles comprising of proteins, nucleic acids and lipids, serve as mediators of cell-cell communication. Gastric cancer tumor-derived exosomes may be involved in the pathological process of peritoneal dissemination by mediating crosstalk between cancer cells and mesothelial cells, to result in the induction of enhanced tumor growth, migratory, adhesive and invasive abilities, peritoneal fibrosis and apoptosis, mesothelial-to-mesenchymal transition, angiogenesis and chemoresistance. The present review focuses on previous studies addressing the exosome-dependent molecular transfer in peritoneal dissemination in gastric cancer and the potential clinical applications.
\end{abstract}

Correspondence to: Professor Li Chen, Department of General Surgery, The Second Affiliated Hospital, College of Medicine, Zhejiang University, 88 Jiefang Road, Hangzhou, Zhejiang 310000, P.R. China

E-mail: 1i-chen@zju.edu.cn

*Contributed equally

Abbreviations: CD, cluster of differentiation; EGFR, epidermal growth factor receptor; EMT, epithelial-to-mesenchymal transition; E-/N-cadherin, epithelial/neural cadherin; HGF, hepatocyte growth factor; HIF-1 $\alpha$, hypoxia inducible factor-1 $\alpha$; HPMC, human peritoneal mesothelial cell; ITG $\beta 1$, integrin $\beta 1$; miRNA/miR, microRNA; MMP, matrix metalloproteinase; MMT, mesothelial-to-mesenchymal transition; siRNA, small interfering RNA; TEX, tumor-derived exosomes; TGF- $\beta 1$, transforming growth factor- $\beta 1$

Key words: exosomes, peritoneal dissemination, gastric cancer, microRNAs

\section{Contents}

1. Introduction

2. Exosomes in peritoneal dissemination of gastric cancer

3. Early detection by exosomal miRNAs

4. Chemoresistance and exosome-based treatment of peritoneal dissemination cases of gastric cancer

\section{Introduction}

Peritoneal dissemination is detected in $14 \%$ of patients with gastric cancer at the time of initial diagnosis, for whom the median survival time is 4 months (1). Gastric cancer patients with peritoneal dissemination cannot undergo radical surgery, and the chemotherapeutic effect is limited due to inadequate distribution of intravenous chemotherapy drugs, blocked by the peritoneal barrier, and tumor chemoresistance (2). The 5 -year survival rate of gastric cancer patients with peritoneal dissemination is only $2 \%$ (3). Peritoneal dissemination is among the most common patterns of recurrence in gastric cancer patients (4). However, it remains unclear how peritoneal dissemination exactly occurs, and there is a need to reveal the underlying molecular mechanism, as well as to develop more effective treatment strategies.

Exosomes are macromolecular, phospholipid bilayer vesicles comprised of proteins, nucleic acids and lipids (5). At 40-120 nm in diameter, exosomes are smaller than other vesicles including microvesicles $(100 \mathrm{~nm}-1 \mu \mathrm{m})$ and apoptotic bodies $(50 \mathrm{~nm}-2 \mu \mathrm{m})(6)$. Exosomes exist in all body fluids and are generated by secretion and budding from various types of cells, including tumor cells (7). They contain key biological molecules including proteins, RNAs and lipids, which are considered to mediate intercellular communication (6). In particular, exosomes deliver proteins and RNAs associated with different pathologies, including neurodegenerative diseases, HIV infection, heart disease and tumor progression, from host cells to recipient cells (8). Tumor-derived exosomes (TEX) may have the capacity to remodel the tumor microenvironment to make it favorable for metastatic niches (7) and, furthermore, may alter the extracellular matrix and attract more cancer cells to the niches (9). Notably, increasing studies indicate that exosomes are involved in the peritoneal dissemination of gastric cancer. The present review focuses on how exosomes participate in 


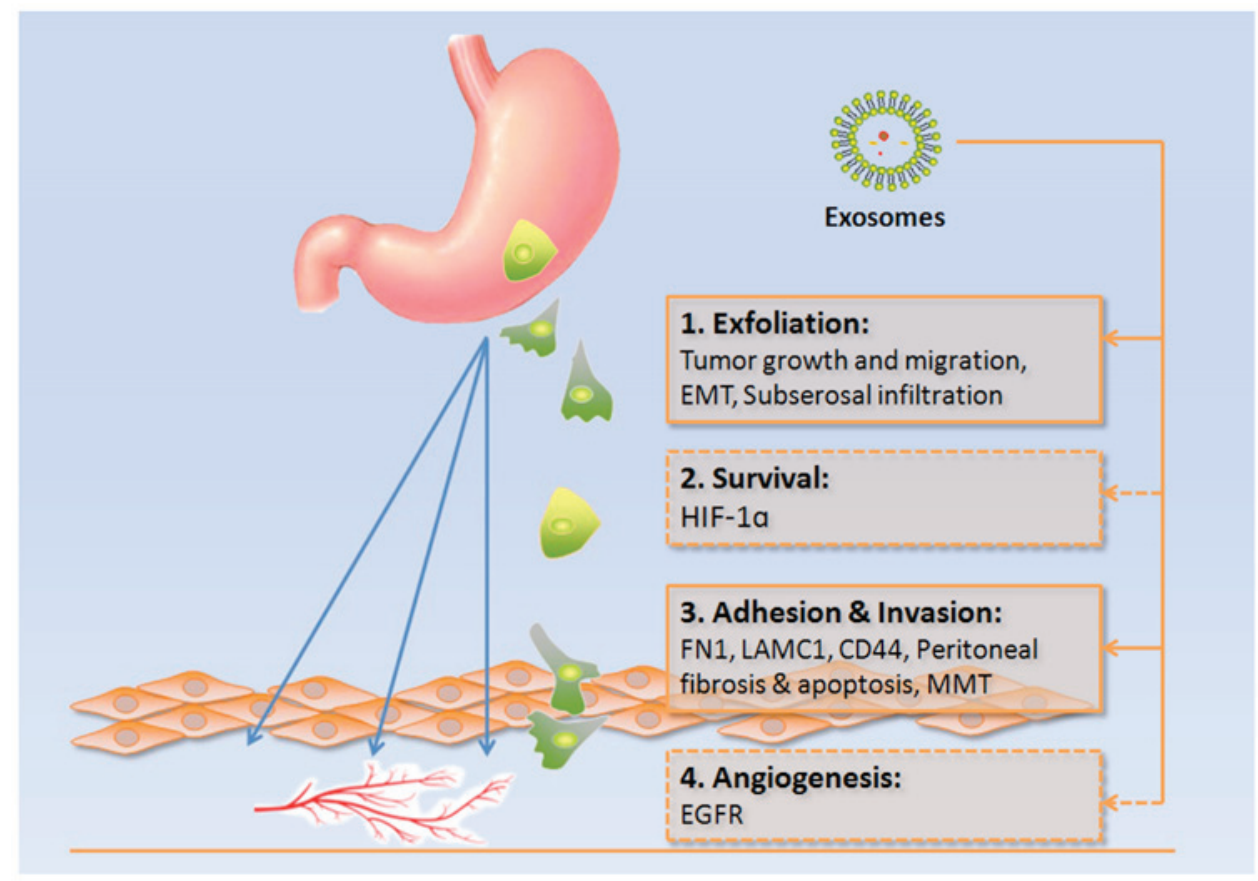

Figure 1. Involvement of exosomes in peritoneal dissemination. Exosome-dependent molecular transfer or signaling pathway activation is crucial in the four stages of peritoneal dissemination of gastric cancer: Exfoliation, survival, adhesion and invasion, and angiogenesis (dotted line box indicates lack of evidence). EMT, epithelial to mesenchymal transition; HIF-1 $\alpha$, hypoxia inducible factor-1 $\alpha$; FN1, fibronectin 1; LAMC1, laminin $\gamma 1$; CD44, cluster of differentiation 44; MMT, mesothelial-to-mesenchymal transition; EGFR, epidermal growth factor receptor.

the pathological process of peritoneal dissemination in gastric cancer, and their potential clinical applications in the future.

\section{Exosomes in peritoneal dissemination of gastric cancer}

In Paget's 'seed and soil' hypothesis, the peritoneum is regarded as the 'soil' and the gastric cancer cells as the 'seeds' (10). In metastatic gastric cancer, direct seeding into the peritoneal cavity occurs in more than $50 \%$ of patients (3). The development of peritoneal dissemination occurs when gastric cancer cells exfoliate from the serosa of the stomach into the abdominal cavity, where they survive before attaching to peritoneal mesothelial cells and invading into the basement membrane to finally induce angiogenesis (3). Exosomes have been revealed to be important mediators of this process, as summarized in Fig. 1 and Table I.

Exfoliation of cancer cells. Previous studies identified that exosomes could promote the proliferation and migration of gastric cancer cells via the phosphatidylinositol-3 kinase/Akt, nuclear factor- $\kappa \mathrm{B}$ and Hedgehog signaling pathways (11-13). Through this exosomal regulation, gastric cancer cells may become more prone to invade the serosa and exfoliate into the cavity with increased proliferative and migratory abilities. Epithelial cells express high levels of epithelial (E)-cadherin, while mesenchymal cells express neural (N)-cadherin, fibronectin and vimentin (14). Dysfunction of E-cadherin has been implicated in gastric cancer progression and may predominantly contribute to invasion of the gastric wall and migration of cancer cells into the free abdominal space (3). Exosomes may induce epithelial-to-mesenchymal transition (EMT), triggering the loss of cell-cell adhesion to facilitate tumor cell invasion and remodeling of the extracellular matrix.
In particular, exosomes derived from gastric cancer have been reported to promote EMT by mediating the transfer of long noncoding RNA ZFAS1 (15). Furthermore, matrix metalloproteinases (MMPs), as the effectors of EMT, have also been identified in exosomes (14). MMP-7 expression has been significantly associated with serosal infiltration and TNM stage in gastric cancer (16). Additionally, Tanaka et al (17) identified that serosal peritoneal mesothelial cells covering the stomach surface could infiltrate the gastric wall to create a novel niche via exosomes containing Wnt family member 3A, as well as guide gastric cancer invasion to the stomach subserosa and facilitate peritoneal metastasis.

Cancer cell survival. Cell-cell interaction is important for cancer cell survival in arduous environments. Protocadherin $\beta$-9 may promote the formation of spheroid colonies of gastric cancer cells, and thus aid the survival of cancer cells in the ascites environment and prevent anoikis and apoptosis induced by chemotherapeutic agents (18). Additionally, intraperitoneal mesenchymal cells may support long-distance migration of disseminated tumor cells by maintaining direct contract, as observed with MKN45 gastric cancer cells (19).

Exosomes exhibit another mechanism of promoting tumor survival through hypoxia inducible factor- $1 \alpha$ (HIF-1 $\alpha$ ), and hypoxia can in turn promote exosome release to increase tumor aggressiveness (20). HIF-1 $\alpha$ itself participates in exosome-mediated pre-metastatic effects in recipient cells. For instance, incubation of naïve breast cancer cells with exosomes shed by hypoxic breast cancer cells promoted focal adhesion, invasion and metastasis (21). HIF-1 $\alpha$ promotes EMT through upregulation of EMT-associated transcription factors, and exosome-mediated delivery of HIF- $1 \alpha$ has been reported to induce changes in the expression of $\mathrm{E}$ - and $\mathrm{N}$-cadherin associated 


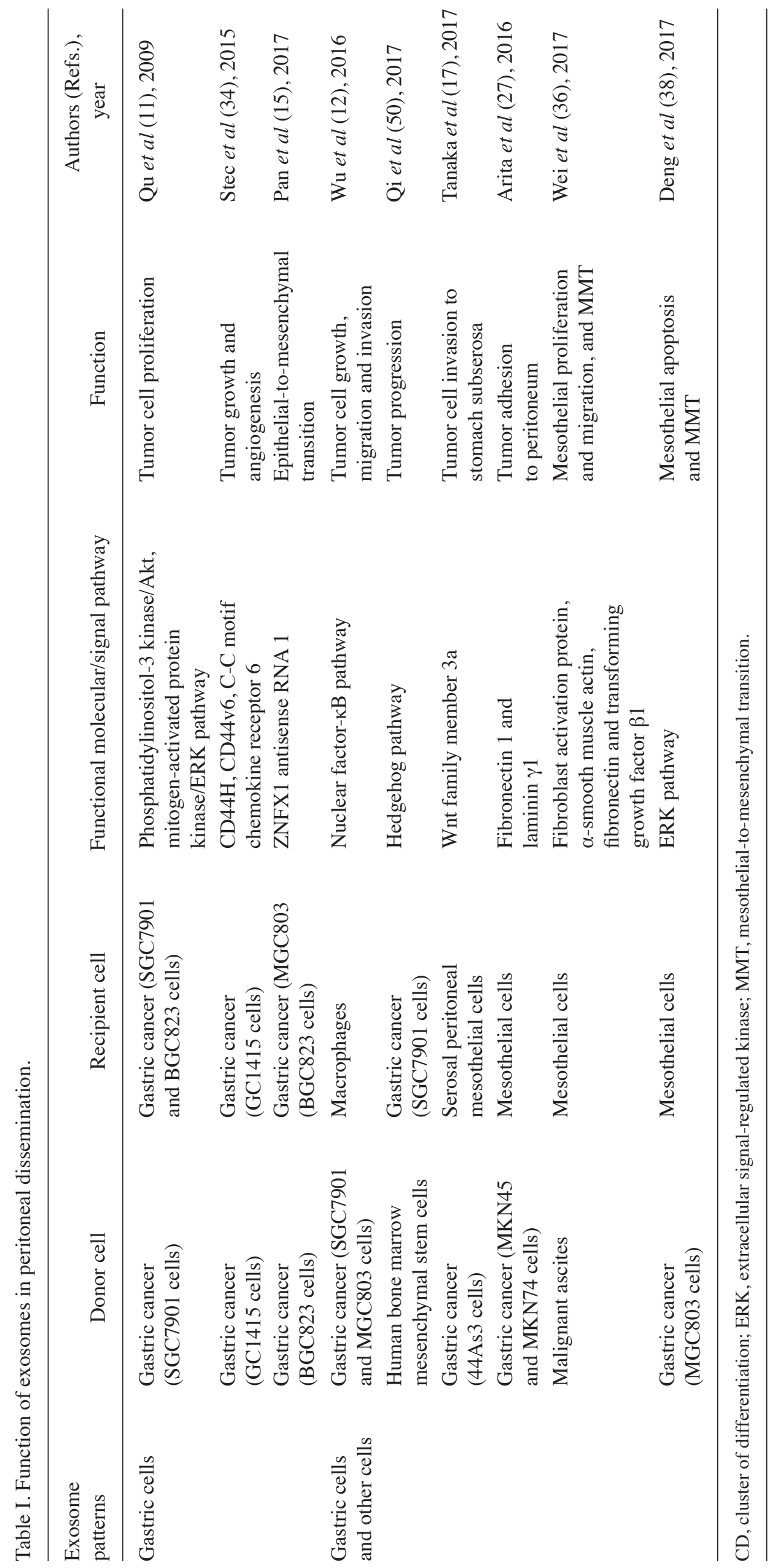


with EMT in nasopharyngeal carcinoma (22). Notably, HIF-1 $\alpha$ expression has been positively correlated with gastric cancer peritoneal dissemination, poor overall and disease-free survival rates, and may be key in the adaptation of cancer cells to the hypoxic microenvironment in the abdominal cavity (23). Gastric cancer stem cells enter and are maintained in the milky spots via the expression and function of HIF-1 $\alpha$ (23). HIF-1 $\alpha$ may increase cancer cell adhesion via integrin $\beta 1$ (ITG $\beta 1$ ). Conversely, dextran sulfate may inhibit peritoneal dissemination of gastric cancer cells through a reduction in HIF-1 $\alpha$ and ITG $\beta 1$ expression (24). At present, it remains to be elucidated whether HIF-1 $\alpha$ is associated with exosomes in peritoneal dissemination.

Adhesion and invasion to mesothelium. The peritoneum is composed of a single layer of flat mesothelial cells and a thin sub-mesothelial connective tissue (25). The mesothelial cells serve as the first barrier against tumor adhesion and invasion (26). If integrity of the barrier is damaged, tumor cells can invade more efficiently into the sub-mesothelial layer (26).

Arita et al (27) cultured mesothelial cells (MeT-5A) and the gastric cancer cell line MKN45 with exosomes derived from MKN45 or MKN74 cells. They observed that TEX were internalized in both mesothelial and gastric cancer cells, which elevated cancer cell migratory ability; furthermore, the expression of the adhesion molecules fibronectin 1 and laminin $\gamma 1$ was upregulated in the Met-5A cells. It was concluded that exosomes may assist gastric cancer cells in adhering to the peritoneum (27).

Cluster of differentiation (CD)44 is the receptor of hyaluronan, expressed on both mesothelial cells and cancer cells. As an adhesive molecule, it is important for cancer cell attachment to the peritoneum (28). The expression of CD44 was identified to be significantly increased in individuals whose gastric lesions progressed along the gastric precancerous cascade infected with Helicobacter pylori (29). Nakashio et al (30) observed that CD44H (standard isoform) and integrins mediate the initial attachment of gastric cancer cells to mesothelial cells, and that increased expression of CD44H promotes early development of peritoneal dissemination. The expression level of CD44 mRNA has also been correlated with peritoneal dissemination (31). CD44 expression is upregulated under the postoperative inflammatory response, which involves elevated levels of reactive oxygen species, transforming growth factor- $\beta 1$ (TGF- $\beta 1$ ), interleukin- $1 \beta$ and tumor necrosis factor- $\alpha$, leading to enhancement of cancer cell adhesion (32). Recently, Nakamura et al (33) co-cultured human peritoneal mesothelial cells (HPMCs) with exosomes derived from ovarian cancer cells and observed that HPMCs, which internalized TEX, exhibited higher level of CD44 expression and underwent mesothelial-to-mesenchymal transition (MMT). Additionally, CD44 overexpression in HPMCs promoted cancer invasion by inducing the HPMCs to secrete MMP9, in order to cross the mesothelial barrier. By contrast, by knockdown of CD44 expression, TEX exerted fewer effects on HPMCs (33). Similarly, isolated exosomes from human gastric cancer cells have demonstrated expression of $\mathrm{CD} 44 \mathrm{H}$ and CD44 variant exon 6 isoforms, which may serve a role in the attachment of TEX to cancer cells (34).

Pathological fluid accumulation, or malignant ascites, in the abdominal cavity, is a result of an imbalance between increased capillary fluid filtration and decreased fluid clearance due to lymphatic obstruction (35). Wei et al (36) investigated the effects of malignant ascite-derived exosomes on the HPMC line HMrSV5. They observed that those exosomes could increase the proliferation and migration of the HPMCs, and induce carcinoma-associated fibroblast transition with overexpression of fibroblast activation protein, $\alpha$-smooth muscle actin and fibronectin. TGF- $\beta 1$ was also identified to be overexpressed in malignant ascites-derived exosomes, which was associated with peritoneal fibrosis. Furthermore, malignant ascite-derived exosomes could regulate xenograft tumor growth in vivo (36). Lv et al (37) confirmed that the expression of TGF- $\beta 1$ was markedly increased during the interaction between gastric cancer cells and peritoneal mesothelial cells, and that inhibition of TGF- $\beta 1$ by P17 peptides could attenuate peritoneal fibrosis and prevent peritoneal dissemination.

Deng et al (38) pretreated nude mice with MGC803 cell-derived exosomes and injected MGC803 cells into the abdominal cavity, and observed that the total weight of the tumor nodules was significantly increased compared with in PBS-treated mice in vivo. Mesothelial cells (HMrSV5) were also cultured with exosomes, which revealed that exosomes induced injury of peritoneal mesothelial cells by promoting apoptosis and MMT, via upregulation of the extracellular signal-regulated kinase pathway (38).

Collectively these previous studies indicate that exosomes may not only facilitate adhesion between gastric cancer cells and mesothelial cells, but also diminish the peritoneal barrier by inducing peritoneal fibrosis, apoptosis and MMT.

Angiogenesis of metastatic cancers. Metastatic cancer cells require high levels of nutrition and oxygen to be supplied through angiogenesis. TEX have been confirmed to be crucial in the angiogenesis of cancers (39-41). TEX induce angiogenesis via transportation of paracrine signaling factors and pro-angiogenic molecules including epidermal growth factor receptor (EGFR) mRNA (42). Zhang et al (43) recently verified that gastric cancer-derived exosomes served an important role in liver metastasis; EGFR levels were enriched in exosomes isolated from the serum of gastric cancer patients but not in healthy subject serum exosomes, and the number of serum exosomes increased with the staging of gastric cancer. In a mouse experiment, it was identified that exosomes of the SGC7901 gastric carcinoma cell line could transport EGFR into the liver and ultimately to the membrane of stromal liver cells. As a consequence, SGC-exosome-derived EGFR activated liver hepatocyte growth factor (HGF) by suppressing expression of the microRNA (miRNA/miR) miR-26a/b, establishing the liver as an ideal site for tumor cell metastasis (43). The EGFR positivity rate of metastatic tumors in patients with peritoneal metastasis is reportedly significantly higher $(70.1 \%)$ than that of metastatic tumors in patients with liver metastasis (37.5\%) (44), which indicates that EGFR expression is associated with peritoneal metastasis. Additionally, a study revealed that angiogenesis was significantly increased when GC1415 cells were co-cultured with TEX for $24 \mathrm{~h}$, as demonstrated by measurement of hemoglobin content (34). However, the phenomenon of angiogenesis induced by TEX was not well explained and requires further investigation. 


\section{Early detection by exosomal miRNAs}

It has been proposed that exosomes may be used as biomarkers of benign diseases. Furthermore, TEX may serve as potent biomarkers for cancer diagnosis, obtained via noninvasive tumor biopsies. At present, circulatory miRNAs are being intensively investigated as biomarkers in different cancers. Research has identified that miRNAs could be partially mediated through exosomes, and exosomal miRNAs have appeared promising as diagnostic molecules for tumors.

Serum exosomal miRNA-105 levels were observed to increase in breast cancer patients upon development of metastatic disease (45). Serum exosomal miR-17-5p and miR-21 were highly expressed in pancreatic adenocarcinoma patients (46). Ren et al (47) analyzed gastric cancer-derived exosomes by next-generation sequencing technology, and demonstrated that these exosomes contained more proteins and RNAs than normal gastric mucosal epithelial cell-derived exosomes, with miRNA-21-5p and miRNA-30a-5p having the most abundant miRNA profiles. Furthermore, six serum miRNAs (miR-10b-5p, miR-132-3p, miR-185-5p, miR-195-5p, miR-20a-3p and miR-296-5p) have been identified as significantly overexpressed in gastric cancer patients compared with in healthy controls (15). Wang et al (48) suggested that serum exosomal miR-19b-3p and miR-106a-5p were novel potential biomarkers for the detection of gastric cancer. Tokuhisa et al (49) suggested that exosomal miR-21 and miR-1225-5p from peritoneal lavage fluid were upregulated in the later stages of gastric cancer and correlated with serosal invasion, which could potentially predict peritoneal recurrence following curative gastric cancer resection (50). However, it remains unknown whether exosomal miRNAs from serum or peritoneum lavage fluid have adequate sensitivity or specificity to serve as novel tumor makers. Another question to address is whether exosomes can differentiate peritoneal dissemination from other metastases including lymph node metastasis. In addition to exosomal miRNAs, other exosomal RNAs and proteins in this aspect require further investigation.

\section{Chemoresistance and exosome-based treatment of peritoneal dissemination cases of gastric cancer}

Accumulating data indicate that exosomes are involved in the chemoresistance of tumors including gastric cancer. For instance, exosomes derived from human mesenchymal stem cells conferred drug resistance in gastric cancer (51). The mechanism of their function appears to be complex. Firstly, TEX may possibly aid cancer cells to survive in the abdominal cavity, promoting tumor invasion and establishment of pre-niches in the sub-mesothelial layer. Furthermore, exosomes may sustain drug resistance by inducing a microenvironment favorable for resistance regarding aspects including EMT phenotype and MDSCs, fibroblast-like cell formation and angiogenesis $(52,53)$. Furthermore, tumor cells may either transfer mRNA/miRNA or receive these molecules from immune cells and stem cells to maintain chemoresistance $(54,55)$.

As an efficient carrier of miRNA, small interfering RNA (siRNA) and engineered drugs, exosome-based treatment is among the most promising anti-tumor therapies. For example, engineered exosomes loaded with doxorubicin can be safely delivered to the tumor microenvironment, demonstrating similar tumor growth inhibition with less adverse effects compared with equipotent free drug (56-58). Recently, Kamerkar et al (59) developed iExosomes (with siRNA or short hairpin RNA targeting KRAS ${ }^{\mathrm{G} 12 \mathrm{D}}$ ) which inhibited metastasis and increased survival in a pancreatic cancer mouse model. Zhang et al (60) demonstrated that exosomes loaded with HGF siRNA could inhibit tumor growth and angiogenesis in gastric cancer. It is hoped that an exosome-based approach for the treatment of gastric cancer with or without peritoneal dissemination will be developed in the near future.

In conclusion, TEX may in part mediate the pathological process of peritoneal dissemination in gastric cancer by mediating crosstalk between cancer cells and mesothelial cells, to result in the induction of enhancements in tumor growth, migratory, adhesive and invasive abilities, peritoneal fibrosis and apoptosis, MMT, angiogenesis and drug resistance. Early diagnosis of peritoneal dissemination by exosomal miRNA detection is emerging as a promising strategy. Furthermore, exosome-based treatment of peritoneal dissemination in gastric cancer may be an effective approach.

\section{Acknowledgements}

The authors would like to thank Dr Gui-Rong Wang at the Department of Surgery, State University of New York Upstate Medical University (Syracuse, NY, USA), for his suggestions and assistance. The authors would also like to thank Mrs Cong-Cong Yu at Zhejiang University (Hangzhou, China), for her support.

\section{Funding}

The present study was supported by the Chinese National Natural Science Fund (grant no. 81372620) and the Zhejiang Provincial Chinese Medicine Scientific Research Fund (grant no. 2017ZA085).

\section{Availability of data and materials}

All data and materials are included in the current review.

\section{Authors' contributions}

$\mathrm{KBC}$, JC and LC designed, wrote and revised the manuscript. $\mathrm{KBC}$ created the figure and table. XLJ, YH and QMS searched for relevant publications and edited the manuscript. All authors read and approved the final version of the manuscript.

\section{Ethics approval and consent to participate}

Not applicable.

\section{Consent for publication}

Not applicable.

\section{Competing interests}

The authors declare no competing interests. 


\section{References}

1. Thomassen I, van Gestel YR, van Ramshorst B, Luyer MD, Bosscha K, Nienhuijs SW, Lemmens VE and de Hingh IH: Peritoneal carcinomatosis of gastric origin: A population-based study on incidence, survival and risk factors. Int J Cancer 134: 622-628, 2014

2. McMullen JRW, Selleck M, Wall NR and Senthil M: Peritoneal carcinomatosis: Limits of diagnosis and the case for liquid biopsy. Oncotarget 8: 43481-43490, 2017.

3. Kanda $\mathrm{M}$ and Kodera $\mathrm{Y}$ : Molecular mechanisms of peritoneal dissemination in gastric cancer. World J Gastroenterol 22: 6829-6840, 2016.

4. Eom BW, Yoon H, Ryu KW, Lee JH, Cho SJ, Lee JY, Kim CG, Choi IJ, Lee JS, Kook MC, et al: Predictors of timing and patterns of recurrence after curative resection for gastric cancer. Dig Surg 27: 481-486, 2010

5. van Dommelen SM, Vader P, Lakhal S, Kooijmans SA, van Solinge WW, Wood MJ and Schiffelers RM: Microvesicles and exosomes: Opportunities for cell-derived membrane vesicles in drug delivery. J Control Release 161: 635-644, 2012.

6. Zaborowski MP, Balaj L, Breakefield XO and Lai CP: Extracellular vesicles: Composition, biological relevance, and methods of study. Bioscience 65: 783-797, 2015.

7. Guo L and Guo N: Exosomes: Potent regulators of tumor malignancy and potential bio-tools in clinical application. Crit Rev Oncol Hematol 95: 346-358, 2015.

8. Isola AL and Chen S: Exosomes: The messengers of health and disease. Curr Neuropharmacol 15: 157-165, 2017.

9. Fujita Y, Yoshioka Y, Ito S, Araya J, Kuwano K and Ochiya T: Intercellular communication by extracellular vesicles and their microRNAs in asthma. Clin Ther 36: 873-881, 2014.

10. Yashiro M, Chung YS, Nishimura S, Inoue $T$ and Sowa $M$ : Fibrosis in the peritoneum induced by scirrhous gastric cancer cells may act as 'soil' for peritoneal dissemination. Cancer 77 (Suppl): 1668-1675, 1996.

11. Qu JL, Qu XJ, Zhao MF, Teng YE, Zhang Y, Hou KZ, Jiang YH, Yang XH and Liu YP: Gastric cancer exosomes promote tumour cell proliferation through PI3K/Akt and MAPK/ERK activation. Dig Liver Dis 41: 875-880, 2009.

12. Wu L, Zhang X, Zhang B, Shi H, Yuan X, Sun Y, Pan Z, Qian H and $\mathrm{Xu}$ W: Exosomes derived from gastric cancer cells activate $\mathrm{NF}-\kappa \mathrm{B}$ pathway in macrophages to promote cancer progression. Tumour Biol 37: 12169-12180, 2016.

13. Qi J, Zhou Y, Jiao Z, Wang X, Zhao Y, Li Y, Chen H, Yang L, $\mathrm{Zhu} \mathrm{H}$ and $\mathrm{Li} \mathrm{Y}$ : Exosomes derived from human bone marrow mesenchymal stem cells promote tumor growth through hedgehog signaling pathway. Cell Physiol Biochem 42: 2242-2254, 2017.

14. Banyard J and Bielenberg DR: The role of EMT and MET in cancer dissemination. Connect Tissue Res 56: 403-413, 2015

15. Pan L, Liang W, Fu M, Huang ZH, Li X, Zhang W, Zhang P, Qian H, Jiang PC, Xu WR and Zhang X: Exosomes-mediated transfer of long noncoding RNA ZFAS1 promotes gastric cancer progression. J Cancer Res Clin Oncol 143: 991-1004, 2017.

16. Chen JQ, Zhan WH, He YL, Peng JS, Wang JP, Cai SR and Ma JP: Expression of heparanase gene, CD44v6, MMP-7 and nm23 protein and their relationship with the invasion and metastasis of gastric carcinomas. World J Gastroenterol 10: 776-782, 2004.

17. Tanaka M, Kuriyama S, Itoh G, Maeda D, Goto A, Tamiya Y, Yanagihara K, Yashiro M and Aiba N: Mesothelial cells create a novel tissue niche that facilitates gastric cancer invasion. Cancer Res 77: 684-695, 2017.

18. Mukai S, Oue N, Oshima T, Imai T, Sekino Y, Honma R, Sakamoto N, Sentani K, Kuniyasu H, Egi H, et al: Overexpression of PCDHB9 promotes peritoneal metastasis and correlates with poor prognosis in patients with gastric cancer. J Pathol 243: 100-110, 2017.

19. Kitayama J, Yamaguchi H, Ishigami H, Matsuzaki K and Sata N: Intraperitoneal mesenchymal cells promote the development of peritoneal metastasis partly by supporting long migration of disseminated tumor cells. PLoS One 11: e0154542, 2016.

20. King HW, Michael MZ and Gleadle JM: Hypoxic enhancement of exosome release by breast cancer cells. BMC Cancer 12: 421, 2012.

21. Wang T, Gilkes DM, Takano N, Xiang L, Luo W, Bishop CJ, Chaturvedi P, Green JJ and Semenza GL: Hypoxia-inducible factors and RAB22A mediate formation of microvesicles that stimulate breast cancer invasion and metastasis. Proc Natl Acad Sci USA 111: E3234-E3242, 2014
22. Aga M, Bentz GL, Raffa S, Torrisi MR, Kondo S, Wakisaka N, Yoshizaki T, Pagano JS and Shackelford J: Exosomal HIF1a supports invasive potential of nasopharyngeal carcinoma-associated LMP1-positive exosomes. Oncogene 33: 4613-4622, 2014.

23. Miao ZF, Wang ZN, Zhao TT, Xu YY, Gao J, Miao F and Xu HM: Peritoneal milky spots serve as a hypoxic niche and favor gastric cancer stem/progenitor cell peritoneal dissemination through hypoxia-inducible factor $1 \alpha$. Stem Cells 32: 3062-3074, 2014.

24. Xu Y, Jin X, Huang Y, Dong J, Wang H, Wang X and Cao X: Inhibition of peritoneal metastasis of human gastric cancer cells by dextran sulphate through the reduction in HIF-1 $\alpha$ and ITG $\beta 1$ expression. Oncol Rep 35: 2624-2634, 2016.

25. Kiyasu Y,Kaneshima S and Koga S: Morphogenesis of peritoneal metastasis in human gastric cancer. Cancer Res 41: 1236-1239, 1981.

26. Mutsaers SE and Wilkosz S: Structure and function of mesothelial cells. Cancer Treat Res 134: 1-19, 2007.

27. Arita T, Ichikawa D, Konishi H, Komatsu S, Shiozaki A, Ogino S, Fujita Y, Hiramoto H, Hamada J, Shoda K, et al: Tumor exosome-mediated promotion of adhesion to mesothelial cells in gastric cancer cells. Oncotarget 7: 56855-56863, 2016.

28. Jayne D: Molecular biology of peritoneal carcinomatosis. Cancer Treat Res 134: 21-33, 2007.

29. Garay J, Piazuelo MB, Majumdar S, Li L, Trillo-Tinoco J, Del Valle L, Schneider BG, Delgado AG, Wilson KT, Correa P and Zabaleta J: The homing receptor CD44 is involved in the progression of precancerous gastric lesions in patients infected with Helicobacter pylori and in development of mucous metaplasia in mice. Cancer Lett 371: 90-98, 2016.

30. Nakashio T, Narita T, Akiyama S, Kasai Y, Kondo K, Ito K, Takagi $\mathrm{H}$ and Kannagi R: Adhesion molecules and TGF-betal are involved in the peritoneal dissemination of NUGC-4 human gastric cancer cells. Int J Cancer 70: 612-618, 1997.

31. Yamamichi K, Uehara Y, Kitamura N, Nakane Y and Hioki K: Increased expression of CD44v6 mRNA significantly correlates with distant metastasis and poor prognosis in gastric cancer. Int $\mathrm{J}$ Cancer 79: 256-262, 1998.

32. Sluiter N, de Cuba E, Kwakman R, Kazemier G, Meijer G and Te Velde EA: Adhesion molecules in peritoneal dissemination: Function, prognostic relevance and therapeutic options. Clin Exp Metastasis 33: 401-416, 2016.

33. Nakamura K, Sawada K, Kinose Y, Yoshimura A, Toda A, Nakatsuka E, Hashimoto K, Mabuchi S, Morishige KI, Kurachi H, et al: Exosomes promote ovarian cancer cell invasion through transfer of CD44 to peritoneal mesothelial cells. Mol Cancer Res 15: 78-92, 2017

34. Stec M, Szatanek R, Baj-Krzyworzeka M, Baran J, Zembala M, Barbasz J, Waligórska A, Dobrucki JW, Mytar B, Szczepanik A, et al: Interactions of tumour-derived micro(nano)vesicles with human gastric cancer cells. J Transl Med 13: 376, 2015.

35. Saif MW, Siddiqui IAR and Sohail MA: Management of ascites due to gastrointestinal malignancy. Ann Saudi Med 29: 369-377, 2009.

36. Wei M, Yang T, Chen X, Wu Y, Deng X, He W, Yang J and Wang Z: Malignant ascites-derived exosomes promote proliferation and induce carcinoma-associated fibroblasts transition in peritoneal mesothelial cells. Oncotarget 8: 42262-42271, 2017.

37. Lv ZD, Zhao WJ, Jin LY, Wang WJ, Dong Q, Li N, Xu HM and Wang HB: Blocking TGF- $\beta 1$ by P17 peptides attenuates gastric cancer cell induced peritoneal fibrosis and prevents peritoneal dissemination in vitro and in vivo. Biomed Pharmacother 88: 27-33, 2017

38. Deng G, Qu J, Zhang Y, Che X, Cheng Y, Fan Y, Zhang S, Na D, Liu Y and Qu X: Gastric cancer-derived exosomes promote peritoneal metastasis by destroying the mesothelial barrier. FEBS Lett 591: 2167-2179, 2017.

39. Jia Y, Chen Y, Wang Q, Jayasinghe U, Luo X, Wei Q, Wang J, Xiong H, Chen C, Xu B, et al: Exosome: Emerging biomarker in breast cancer. Oncotarget 8: 41717-41733, 2017.

40. Pant S, Hilton $\mathrm{H}$ and Burczynski ME: The multifaceted exosome: Biogenesis, role in normal and aberrant cellular function, and frontiers for pharmacological and biomarker opportunities. Biochem Pharmacol 83: 1484-1494, 2012.

41. Azmi AS, Bao B and Sarkar FH: Exosomes in cancer development, metastasis, and drug resistance: A comprehensive review. Cancer Metastasis Rev 32: 623-642, 2013.

42. Steinbichler TB, Dudás J, Riechelmann H and Skvortsova II: The role of exosomes in cancer metastasis. Semin Cancer Biol 44: 170-181, 2017. 
43. Zhang H, Deng T, Liu R, Bai M, Zhou L, Wang X, Li S, Wang X, Yang $\mathrm{H}, \mathrm{Li} \mathrm{J}$, et al: Exosome-delivered EGFR regulates liver microenvironment to promote gastric cancer liver metastasis. Nat Commun 8: 15016, 2017.

44. Saito T, Nakanishi H,Mochizuki Y, Ito S, Ito Y, Misawa K, Yatabe Y, Yamamichi $\mathrm{K}$ and Kondo E: Preferential HER2 expression in liver metastases and EGFR expression in peritoneal metastases in patients with advanced gastric cancer. Gastric Cancer 18: 711-719, 2015.

45. Zhou W, Fong MY, Min Y, Somlo G, Liu L, Palomares MR, Yu Y Chow A, O'Connor ST, Chin AR, et al: Cancer-secreted miR-105 destroys vascular endothelial barriers to promote metastasis. Cancer Cell 25: 501-515, 2014.

46. Que R, Ding G, Chen J and Cao L: Analysis of serum exosomal microRNAs and clinicopathologic features of patients with pancreatic adenocarcinoma. World J Surg Oncol 11: 219, 2013.

47. Ren J, Zhou Q, Li H, Li J, Pang L, Su L, Gu Q, Zhu Z and Liu B: Characterization of exosomal RNAs derived from human gastric cancercells by deep sequencing. Tumour Biol 39: 1010428317695012, 2017.

48. Wang N, Wang L, Yang Y, Gong L, Xiao B and Liu X: A serum exosomal microRNA panel as a potential biomarker test for gastric cancer. Biochem Biophys Res Commun 493: 1322-1328, 2017.

49. Tokuhisa M, Ichikawa Y, Kosaka N, Ochiya T, Yashiro M, Hirakawa K, Kosaka T, Makino H, Akiyama H, Kunisaki C and Endo I: Exosomal mirnas from peritoneum lavage fluid as potential prognostic biomarkers of peritoneal metastasis in gastric cancer. PLoS One 10: e0130472, 2015.

50. Qi J, Zhou Y, Jiao Z, Wang X, Zhao Y, Li Y, Chen H, Yang L, $\mathrm{Zhu} \mathrm{H}$ and $\mathrm{Li} \mathrm{Y}$ : Exosomes derived from human bone marrow mesenchymal stem cells promote tumor growth through hedgehog signaling pathway. Cell Physiol Biochem 42: 2242-2254, 2017.

51. Ji R, Zhang B, Zhang X, Xue J, Yuan X, Yan Y, Wang M, Zhu W, Qian $\mathrm{H}$ and $\mathrm{Xu} \mathrm{W}$ : Exosomes derived from human mesenchyma stem cells confer drug resistance in gastric cancer. Cell Cycle 14: 2473-2483, 2015.
52. Naito Y, Yoshioka Y, Yamamoto Y and Ochiya T: How cancer cells dictate their microenvironment: Present roles of extracellular vesicles. Cell Mol Life Sci 74: 697-713, 2017.

53. Gopal SK, Greening DW, Rai A, Chen M, Xu R, Shafiq A, Mathias RA, Zhu HJ and Simpson RJ: Extracellular vesicles: Their role in cancer biology and epithelial-mesenchymal transition. Biochem J 474: 21-45, 2017.

54. Lobb RJ, van Amerongen R, Wiegmans A, Ham S, Larsen JE and Möller A: Exosomes derived from mesenchymal non-small cell lung cancer cells promote chemoresistance. Int J Cancer 141: 614-620, 2017.

55. Zheng P, Chen L, Yuan X, Luo Q, Liu Y, Xie G, Ma Y and Shen L: Exosomal transfer of tumor-associated macrophage-derived miR-21 confers cisplatin resistance in gastric cancer cells. J Exp Clin Cancer Res 36: 53, 2017.

56. Toffoli G,Hadla M, Corona G, Caligiuri I,Palazzolo S, Semeraro S, Gamini A, Canzonieri V and Rizzolio F: Exosomal doxorubicin reduces the cardiac toxicity of doxorubicin. Nanomedicine (Lond) 10: 2963-2971, 2015.

57. Gomez-Cabrero A, Wrasidlo W and Reisfeld RA: IMD-0354 targets breast cancer stem cells: A novel approach for an adjuvant to chemotherapy to prevent multidrug resistance in a murine model. PLoS One 8: e73607, 2013.

58. Jang SC, Kim OY, Yoon CM, Choi DS, Roh TY, Park J, Nilsson J, Lötvall J, Kim YK and Gho YS: Bioinspired exosome-mimetic nanovesicles for targeted delivery of chemotherapeutics to malignant tumors. ACS Nano 7: 7698-7710, 2013.

59. Kamerkar S, LeBleu VS, Sugimoto H, Yang S, Ruivo CF, Melo SA, Lee J and Kalluri R: Exosomes facilitate therapeutic targeting of oncogenic KRAS in pancreatic cancer. Nature 546: 498-503, 2017.

60. Zhang H, Wang Y, Bai M, Wang J, Zhu K, Liu R, Ge S, Li J, Ning T, Deng T, et al: Exosomes serve as nanoparticles to suppress tumor growth and angiogenesis in gastric cancer by delivering hepatocyte growth factor siRNA. Cancer Sci 109: 629-641, 2018. 